\title{
SOME QUESTIONS CONCERNING ALTERNATIVE RINGS
}

\author{
M. F. SMILEY
}

1. Introduction. Our purpose is to summarize our present knowledge of alternative rings in the case in which no a priori finiteness assumptions are made and to indicate a number of problems in this field. One defines an alternative ring by replacing the law $a(b c)=(a b) c$ in the definition of an associative ring by the laws $a(a b)=a^{2} b$ and $(a b) b=a b^{2}$. The name is derived from the fact that the associator $(a, b, c)=(a b) c-a(b c)$ is an alternating function of its arguments. The name as well as much of our knowledge of the finite-dimensional case is due to M. Zorn [52-55], 1 although N. Jacobson [25], A. A. Albert [1], R. D. Schafer [41-43], and Dubisch and Perlis [19] have also contributed.

We shall use the terms ring and algebra in place of nonassociative ring and nonassociative algebra. If the nonzero elements of a ring form a loop [4] under multiplication (that is, if each pair of elements in the equation $a b=c$ uniquely determines the remaining element and a unit element 1 is present), we call the ring a division ring [cf. $2 ; 16$ ]. The center $[2 ; 26]$ of a ring $A$ consists of those elements $c$ in $A$ for which $c x=x c$ and $(c x) y=c(x y)=x(c y)$ for every $x$ and $y$ in $A$. When the center of $A$ is a division ring, then $A$ is a vector space over its center and we call the dimension of this vector space the dimension of $A$.

We divide our discussion into three parts wherein the primary interest is geometric, algebraic, and topological, respectively.

2. Geometry. Ruth Moufang [33-38] was the first to derive the geometric meaning of the alternative law as a weak form of Desargues' Theorem in plane projective geometry. Marshall Hall, Jr. [22-23] has given a new proof which is mainly algebraic and which avoids assumptions concerning order or characteristic in the plane. In its affine form, the theorem used by Hall merely asserts that if corresponding vertices of two triangles are on parallel lines, while two pairs of corresponding sides are parallel, then the remaining sides are also parallel. The works of Moufang and of Hall suggest the following questions.

1. Is there an ordered alternative division ring which is not associative? (Moufang [38])

A thirty minute address delivered before the East Lansing Meeting of the Society on February 24, 1950, by invitation of the Committee to Select Hour Speakers for Western Sectional Meetings; received by the editors March 29, 1950.

${ }^{1}$ Numbers in brackets denote references given at the end of the paper. 
2. Is every isotope [2] with unit of an alternative division ring really an isomorph? (Hall [22])

3. Is there a division ring for which the identity $a(a b)=a^{2} b$ holds but $(a b) b=a b^{2}$ fails?

These questions have been answered by Zorn [53], Schafer [41], and Albert [9], respectively no, yes, and no, ${ }^{2}$ in the finite-dimensional case. R. H. Bruck has shown that a natural generalization of Hilbert's procedure fails to answer the first question [16]. Albert ${ }^{3}$ has remarked that the analogous question for Jordan rings [6] has a very easy answer for we may set $2(x \cdot y)=x y+y x$ in any ordered associative but not commutative ring $A$ to obtain an ordered Jordan ring $A_{+}$, provided that the positive elements of $A_{+}$coincide with those of $A$.

Since configuration theorems in plane projective geometry are reflected in algebraic identities in the coördinate ring, we are led to consider these next. Kaplansky [29] has given a sweeping generalization of a result of Hall [22] by showing that a primitive ring [27] which satisfies a polynomial identity is finite-dimensional. An important question (no. 4) is how this may be generalized to alternative rings. For the case of an alternative algebraic division algebra of degree two, Albert [8] has given an affirmative answer. (As noted by Jacobson [29], every alternative algebraic algebra of bounded degree satisfies a polynomial identity.) We have given [49] an independent proof of Albert's result (including the case of characteristic two, avoided by Albert), starting from the appropriate identity $\left[[x, y]^{2}, z\right]=0$ of Hall $[22]$. The identity $x y=y x$ in an alternative division ring implies the associative law, ${ }^{4}$ as Bruck has proved by a simple algebraic argument [46], and whose geometric counterpart in the form of the Theorem of Pappus and the weakened Desargues Theorem seems to be well known [40]. In fact, a commutative alternative ring with zero radical (see $\$ 3$ ) is associative, but commutative alternative but not associative rings exist [47]. Nothing further seems to be known about this question. The identity attributed by Kaplansky to Kolchin [29; cf. 51, p. 98] for associative algebras of finite dimension applies to algebras if we use a fixed association in each of its terms. This indicates that an algebra of finite dimension is not completely nonassociative since, for example,

${ }^{2}$ At least when the characteristic is zero.

${ }^{3}$ This remark of Albert was mentioned by the discussant, Saunders MacLane.

${ }^{4}$ It is interesting to note that M. H. A. Newman (A characterization of Boolean algebras and rings, J. London Math. Soc. vol. 16 (1941) pp. 256-272, and Relatively complemented algebras, J. London Math. Soc. vol. 17 (1942) pp. 34-47) observed that the rule $(a b) b=a(b b)$ was sufficient to ensure the associativity of his generalized Boolean rings. 
algebras of dimension two satisfy the identity $x(y z)+y(z x)+z(x y)$ $-x(z y)-y(x z)-z(y x)=0$. A similar remark applies to an arbitrary binary operation defined on a finite set.

Let us finally mention the following extension of a theorem of $\mathrm{H}$. Cartan [18] which is due to $\mathrm{R}$. Brauer [12]. If $B$ is a normal division subring of a division ring $A$, then $B=A$ or $B$ is contained in the center of $A$. We use the term normal as it applies to the subloop of nonzero elements of $B[4 ; 5 ; 11 ; 17 ; 21 ; 45]$ and center as defined in $\S 1$. The additiona $1^{5}$ requirements on the elements of $B$, namely, that $b(x y)$ $=(b x) y=y(b y)$ for every $x, y \in A$ and $b \in B$ when $B \neq A$, are easily proved using Brauer's method. L. K. Hua [24] has also given an elementary proof of Cartan's theorem which is not greatly different from Brauer's. However, it is interesting to observe that several of the related results stated by $\mathrm{Hua}$ in [24] are also valid in alternative division rings. In particular, the conjugates $x^{-1}$ ax of an element $a$ of an alternative division ring $A$ are all equal to $a$ or they generate $A$. This follows from Hua's proof and the fact that $3(a, x, y)=(a, x, y)$ $-(x, a, y)+(x, y, a)=0$ for every $x, y \in A$, provided that $a z=z a$ for every $z \in A$. When $3 \neq 0$, the center of $A$ coincides with the set of all $a$ satisfying $a z=z a$ for every $z \in A$. But when $3=0$, this question is unanswered, and we may assert Theorems 1-8 of Hua [24] for alternative division rings only if we replace the word "center" by the words "the set of elements $a \in A$ for which $a z=z a$ for every $z \in A$."

3. Algebra. The structure theory of associative rings presented ${ }^{6}$ by Jacobson $[26-27 ; 10]$ is so elegant that its generalization to rings has attracted the interest of several writers. In fact, Dubisch and Perlis were aware in 1943 (prior to the publication of this theory) that the set of all properly nilpotent elements of an alternative algebra (that is, the radical in the sense of Zorn [55]) could be characterized as what is now called the Perlis-Jacobson radical: ${ }^{7}$

$$
R=\left[a ; b \in(a)_{r} \text { implies that }(1-b) A=A\right] .
$$

Our observation that the proof of Forsythe and McCoy [20] that an associative regular ring without nonzero nilpotent elements is a subdirect sum [32] of associative division rings is easily extendable to alternative rings [46] led us to study this problem and we were able to show that the Perlis-Jacobson radical of an alternative ring is an

5 That is, those not already proved in [12].

${ }^{6}$ Cf. J. Dieudonné, Sur le socle d'un anneau et les anneaux simple infinis, Bull. Soc. Math. France vol. 70 (1942) pp. 46-75.

${ }^{7}$ Dubisch and Perlis [19]. In this section we shall use $(a)_{r}$ and $(a)$ to denote the right ideal and the ideal, respectively, generated by $a$. We shall also use the abbreviation $(1-b) A$ for the set of elements $[x-b x ; x \in A]$. 
ideal [47]. Brown and McCoy offered a variant of the Perlis-Jacobson radical [14] for associative rings which we have shown to be applicable to arbitrary rings [48] and which reduces to Zorn's radical of an alternative ring when his chain conditions hold [55].

A connection of the Perlis-Jacobson radical of an alternative ring with a subdirect decomposition [32] of the alternative ring is still lacking in the general case, although we have succeeded in exhibiting such a connection for an alternative ring $A$ (which we call special) in which the set $(1-a) A$ is a right ideal of $A$ for every $a$ in $A$ [50]. Under this assumption, we may prove as in the associative case that $R \geqq \cap(M: A)$, where $M$ ranges over the $\operatorname{modular}^{8}$ maximal right ideals of $A$ and $(M: A)=[a ; x a \in I$ for every $x \in A]$ is the quotient ideal of $M$. To secure $R \leqq \bigcap(M: A)$, we consider an element $a$ not in $\cap(M: A)$ and hence not in some modular maximal right ideal $M$. Then $A$ is the sum of $M$ and $(a)_{r}$. In particular, the left unit $e$ of $A$ modulo $M$ has the form $e=m+b$, with $m$ in $M$ and $b$ in $(a)_{r}$. But then $(1-b) x=x-(e-m) x=x-e x+m x$ is in $M$ for every $x$ in $A$ and $a$ cannot be in $R$. Thus $R=0$ entails $\cap(M: A)=0$ and a consequent representation of $A$ as a subdirect sum of special alternative rings which are primitive in Jacobson's sense; each summand possesses a modular maximal right ideal whose quotient ideal is zero. One may easily prove the converse statement that a subdirect sum of primitive special alternative rings has zero Perlis-Jacobson radical.

Brown and McCoy have discovered a generalization of the PerlisJacobson radical which applies to arbitrary rings [15] and which Brown has associated with a subdirect decomposition of the ring ${ }^{9}$ [13]. They define $N_{r}$ as the set of all elements $a$ of a ring $A$ for which every element $b$ in $(a)$ is such that the right ideal generated by the set $(1-b) A$ is $A$. They then show that $N_{r}$ is an ideal of $A$. Brown [13] replaces Jacobon's quotient ( $I: A)$ of a right ideal $I$ of $A$ by the largest ideal $I^{\prime}$ contained in $A$. It should be noted that for a modular right ideal $I$, we have $I^{\prime}=(I: A)$ provided that $(I: A)$ is an ideal of $A$. Brown then proves that $N_{r}=\cap M^{\prime}$ as $M$ ranges over the modular maximal right ideals of $A$ by a slight modification of the argument of the preceding paragraph. This argument yields another proof that $N_{r}$ is an ideal. The result also shows that $R=N_{r}$ for special alternative rings, but the question of the validity of this equation for arbitrary alternative rings is open. When $N_{r}=0$, we see that $A$ is a subdirect

${ }^{8} \mathrm{~A}$ right ideal $I$ is modular in case there is an element $e \in A$ such that $(1-e) A \leqq I$, that is, $e$ is a left unit for $A$ modulo $I$. Segal [44] uses the term regular, but we prefer the present terminology of Jacobson.

${ }^{9}$ We are indebted to Brown and to McCoy for a stimulating correspondence in the source of which we were informed of some of their most recent results. 
sum of rings each of which is (right-) primitive in the sense that it contains a modular maximal right ideal $M$ with $M^{\prime}=0$. The duality of the Perlis-Jacobson radical of an alternative ring with respect to interchange of right and left multiplications is lost in this process of generalization as a simple example shows. By use of the theory of normal subloops of a loop $[5 ; 11 ; 17 ; 45]$, we may dispense with the associativity (as well as the commutativity) of addition ${ }^{10}$ provided that we interpret $(1-b) A$ as the set $[x+b y ; x \in A, x+y=0]$. The distributive laws and associativity of addition enforce, however, the commutativity of addition when $N_{r}=0$. It is possible to phrase this theory of Brown and McCoy in the setting of groups with operators [15]. A consequence of this more general formulation is the fact, proved by Brown and McCoy in [15], that every alternative ring has a greatest regular ideal. ${ }^{11}$

The results of Brown and McCoy and of Brown suggest a host of questions which will readily occur to the reader. One should note especially that nil rings are automatically placed in the limbo of radical rings. The vital question (no. 5) seems to be: "What is the nature of primitive alternative rings?" For associative rings, Jacobson shows that a primitive ring is a dense ring of linear transformations in a vector space over a division ring. All that seems to be known in the alternative case is that if the modular maximal right ideal $M$ contains every associator, then $A$ is associative.

One of the main difficulties in the study of alternative rings is the lack of some sort of representation closely connected with an associative ring. Saunders MacLane has suggested (in conversation) that there is a cohomology theory of alternative rings (question no. 6) and perhaps such a theory might be helpful in the study of primitive alternative rings.

In concluding this section, we mention the so-called Theorem of Artin which states that every two elements of an alternative ring generate an associative subring [52]. R. Moufang proved a generalization for alternative division rings: if $(a, b, c)=0$, then $a, b, c$ generate an associative division subring [39]. The analogous statement is true in an arbitrary alternative ring [47], and no doubt still more general formulations are possible. ${ }^{12}$

${ }^{10}$ It is possible to have a finite system of this kind in which the nonzero elements form a commutative group under multiplication but in which addition is not associative. (See Neofields by L. J. Paige, Duke Math. J. vol. 16 (1949) pp. 39-60.)

11 A subset $S$ of an alternative ring $A$ is regular (in von Neumann's sense) in case every $a \in S$ has the form $a x a$ with $x \in A$.

12 (Added July 12,1950.) Since this was written, R. H. Bruck has shown by a remarkably simple argument that a subset $S$ of an alternative ring $A$ which satisfies 
4. Topology. We turn finally to topology as a method of studying alternative rings. Here the main results are those of Albert [7-8] and of Jacobson and Taussky [28]. Albert assumes that his $\operatorname{ring} A$ is algebraic relative to the real field and has a homogeneous valuation. He then shows that $A$ is alternative and applies his result, mentioned in $\$ 2$, to show that $A$ is associative (and hence already characterized) or the Cayley numbers. In his discussion of the results of Jacobson and Taussky, Kaplansky [31] also obtains the Cayley numbers as the only not associative alternative division ring which is both connected and locally connected, and he conjectures that a similar result holds in the totally disconnected, locally compact case. It seems likely that topological methods will yield further results. In fact, many of the preliminary results of Kaplansky in [30] are valid at least for special alternative rings. A difficulty appears in the proof that group neighborhoods contain ideal neighborhoods in the bounded case. We may obtain this result if we strengthen the definition of (right-) bounded ring $A$ to: "For every neighborhood $U$ of 0 , there is a neighborhood $V$ such that $V \rho \leqq U$ for every product $\rho$ of finitely many right multiplications of $A$." (This is automatically satisfied in a right bounded associative ring.) But then it seems difficult to prove that a compact ring is bounded. However, we can show that a compact and bounded alternative ring with zero Perlis-Jacobson radical is isomorphic and homeomorphic to a Cartesian direct sum of finite simple alternative rings. This subsumes the corresponding result of $\mathrm{Ka}$ plansky in [30]. By a result of Zorn [52], we see that the only not associative summands permitted are merely finite Cayley-Dickson algebras with divisors of zero.

Added in proof, (January 5, 1951). At the International Congress of Mathematicians 1950, R. H. Bruck announced that he and Erwin Kleinfeld have proved that an alternative division ring in which $2 \neq 0$ is either associative or is a Cayley-Dickson algebra over its center. This outstanding result answers a number of questions suggested in this paper. Thus, aside from the limitation on the characteristic, Questions 1 and 2, as well as the conjecture of Kaplansky mentioned in $\$ 4$, are settled.

\section{REFERENCES}

1. A. A. Albert, Quadratic forms permitting composition, Ann. of Math. vol. 43 (1942) pp. 161-177.

$(x, y, z)=0$ for $x, y, z \in S$ generates an associative subring provided that $A$ is commutative or satisfies " $3(a, b, c)=0$ for $a, b, c \in A$ implies that $(a, b, c)=0$." We have given an example of an alternative algebra $A$ of dimension 65 over the field of three elements which is generated by a set $S$ of four elements and which is not associative although $(x, y, z)=0$ for $x, y, z \in S$. 
2. - Non-associative algebras I: Fundamental concepts and isotopy, Ann. of Math. vol. 43 (1942) pp. 685-708.

3. - The radical of a non-associative algebra, Bull. Amer. Math. Soc. vol. 48 (1942) pp. 891-897.

4. - Quasigroups. I, Trans. Amer. Math. Soc. vol. 54 (1943) pp. 507-519.

5. - Quasigroups. II, Trans. Amer. Math. Soc. vol. 55 (1944) pp. 401-419.

6. - On Jordan algebras of linear transformations, Trans. Amer. Math. Soc. vol. 59 (1946) pp. 524-555.

7. - Absolute valued real algebras, Ann. of Math. vol. 48 (1947) pp. 495-501.

8. - Absolute-valued algebraic algebras, Bull. Amer. Math. Soc. vol. 55 (1949) pp. 763-768.

9. - On right alternative algebras, Ann. of Math. vol. 50 (1949) pp. 318-328.

10. E. Artin, The influence of J.H. M. Wedderburn on the development of modern algebra, Bull. Amer. Math. Soc. vol. 56 (1950) pp. 65-72.

11. R. Baer, The homomorphism theorems for loops, Amer. J. Math. vol. 67 (1945) pp. $450-460$.

12. R. Brauer, On a theorem of H. Cartan, Bull. Amer. Math. Soc. vol. 55 (1949) pp. 619-620.

13. B. Brown, An extension of the Jacobson radical, to appear in the Proceedings of the American Mathematical Society.

14. B. Brown and N. H. McCoy, Radicals and subdirect sums, Amer. J. Math. vol. 69 (1947) pp. 46-58.

15. - Some theorems on groups with applications to ring theory, Trans. Amer. Math. Soc. vol. 69 (1950) pp. 302-311.

16. R. H. Bruck, Some results in the theory of linear non-associative algebras, Trans. Amer. Math. Soc. vol. 56 (1944) pp. 141-199.

17. - Contributions to the theory of loops, Trans. Amer. Math. Soc. vol. 60 (1946) pp. 245-354.

18. H. Cartan, Théorie de Galois pour les corps non commutatifs, Annales Scientifiques de l'École Normale Supérieure (3) vol. 64 (1947) pp. 59-77.

19. R. Dubisch and S. Perlis, On the radical of a non-associative algebra, Amer. J. Math. vol. 70 (1948) pp. 540-546.

20. A. Forsythe and N. H. McCoy, On the commutativity of certain rings, Bull. Amer. Math. Soc. vol. 52 (1946) pp. 523-526.

21. G. N. Garrison, Quasigroups, Ann. of Math. vol. 41 (1940) pp. 474-487.

22. M. Hall, Projective planes, Trans. Amer. Math. Soc. vol. 54 (1943) pp. 229277.

23. - Correction to "Projective planes," Trans. Amer. Math. Soc. vol. 65 (1949) p. 474.

24. L. K. Hua, Some properties of a field, Proc. Nat. Acad. Sci. U. S. A. vol. 35 (1949) pp. 533-537.

25. N. Jacobson, Cayley numbers and normal simple Lie algebras of type $G$, Duke Math. J. vol. 5 (1939) pp. 775-783.

26. - Structure theory of simple rings without finiteness assumptions, Trans. Amer. Math. Soc. vol. 57 (1945) pp. 228-245.

27. - The radical and semi-simplicity for arbitrary rings, Amer. J. Math. vol. 67 (1945) pp. 300-320.

28. N. Jacobson and O. Taussky, Locally compact rings, Proc. Nat. Acad. Sci. U. S. A. vol. 21 (1931) pp. 106-108.

29. I. Kaplansky, Rings with a polynomial identity, Bull. Amer. Math. Soc. vol. 54 (1948) pp. 575-580. 
30. - Topological rings, Amer. J. Math. vol. 69 (1947) pp. 153-183.

31. - Topological rings, Bull. Amer. Math. Soc. vol. 54 (1948) pp. 809-826.

32. N. H. McCoy, Subdirect sums of rings, Bull. Amer. Math. Soc. vol. 53 (1947) pp. 856-877.

33. R. Moufang, Zur Struktur der projectiven Geometrie der Ebene, Math. Ann. vol. 105 (1931) pp. 536-601.

34. - Die Einfilhrung der idealen Elemente in die ebene Geometrie mit Hilfe des Sätzes vom vollständigen Vierseit, Math. Ann. vol. 105 (1931) pp. 759-778.

35. - Die Schnittpunktsätze des projectiven speziellen Fïnfecksnetzes in ihrer Abhängigkeit voneinander (Das A-Netz), Math. Ann. vol. 106 (1932) pp. 755-795.

36. - Ein Satz über die Schnittpunktsätze des allgemeinen Fünfecksnetze [Das (A, B)-Netz], Math. Ann. vol. 107 (1932) pp. 124-139.

37. - Die Desarguesschen Sätze vom Rang 10, Math. Ann. vol. 108 (1933) pp. 296-310.

38. - Alternativkörper und der Satz vom vollständigen Vierseit $\left(D_{9}\right)$, Abh. Math. Sem. Hamburgischen Univ. vol. 9 (1933) pp. 207-222.

39. ——, Zur Struktur von Alternativkörpern, Math. Ann. vol. 110 (1935) pp. 416-430.

40. K. Reidemeister, Grundlagen der Geometrie, Berlin, 1930.

41. R. D. Schafer, Alternative algebras over an arbitrary field, Bull. Amer. Math. Soc. vol. 49 (1943) pp. 549-555.

42. - The Wedderburn principal theorem for alternative algebras, Bull. Amer. Math. Soc. vol. 55 (1949) pp. 604-614.

43. - Inner derivations of non-associative algebras, Bull. Amer. Math. Soc. vol. 55 (1949) pp. 769-776.

44. I. Segal, The group algebra of a locally compact group, Trans. Amer. Math. Soc. vol. 61 (1947) pp. 69-105.

45. M. F. Smiley, An application of lattice theory to quasigroups, Bull. Amer. Math. Soc. vol. 50 (1944) pp. 782-786.

46. - Alternative regular rings without nilpotent elements, Bull. Amer. Math. Soc. vol. 53 (1947) pp. 775-778.

47. - The radical of an alternative ring, Ann. of Math. vol. 49 (1948) pp. $702-709$.

48. - An application of a radical of Brown and McCoy to non-associative rings, Amer. J. Math. vol. 72 (1950) pp. 93-100.

49. - A remark on a theorem of Marshall Hall, Proceedings of the American Mathematical Society vol. 1 (1950) pp. 342-343.

50. - Special alternative rings (abstract), Bull. Amer. Math. Soc. vol. 55 (1949) p. 1051.

51. H. Weyl, The classical groups, 2d ed., Princeton, 1946.

52. M. Zorn, Theorie der alternativen Ringe, Abh. Math. Sem. Hamburgischen Univ. vol. 8 (1930) pp. 123-147.

53. - Alternativkörper und quadratische Systeme, Abh. Math. Sem. Hamburgischen Univ. vol. 9 (1933) pp. 395-402.

54. - The automorphisms of Cayley's non-associative algebra, Proc. Nat. Acad. Sci. U. S. A. vol. 21 (1935) pp. 355-358.

55. - Alternative rings and related questions. I: Existence of the radical, Ann. of Math. vol. 42 (1941) pp. 676-686.

State University OF Iowa 